ihren Personalschlüssel verloren. Versorgungs- und Bearbeitungslücken lassen sich nicht fundiert skandalisieren, notwendige rechtspolitische Entwicklungen gestalten sich schwieriger. Auch zeigt sich Deutschland durch das Fehlen einer einheitlichen Datengrundlage aus auch international vergleichbaren Parametern in der weit über die Ländergrenzen hinausgehenden fachpolitischen Diskussion als nicht anschlussfähig.

Der djb mahnt daher in der Rechtspolitik und bei den Praktiker/inne/n an, intra- wie interdisziplinär eine verbindliche gemeinsame begriffliche Arbeitsgrundlage zu schaffen, die international anschlussfähig ist. Diese ist in alle von Justiz und Polizei geführten Statistiken zu integrieren. Zudem sind diese Statistiken dann, soweit nicht - wie in der polizeilichen Statistik - schon teilweise verwirklicht, im Hinblick auf den Bereich der häuslichen Gewalt auszudifferenzieren und damit aussagekräftiger zu gestalten.

Vernetzung auf lokaler, regionaler Landes- und Bundesebene ist ein grundlegender Baustein einer gelingenden Bekämpfung der häuslichen Gewalt in der Gesellschaft. In allen Bundesländern ist hier bereits viel geleistet worden. Einzubeziehende Professionen sind aber über den Kreis von Polizei, Staatsanwaltschaft und Opferunterstützungseinrichtungen hinaus der Kinder- und Jugendbereich, Gesundheitsämter und Ärzt/inn/e/n sowie der Schulbereich.

Für den djb ist es wichtig, überall nicht nur interventiv, sondern verstärkt auch präventiv, zum Beispiel in den Berei- chen Schule, Freizeit, Arbeits- und Wohnsituation die Problematik häuslicher Gewalt und die Notwendigkeit ihrer Bekämpfung deutlich zu machen. Hier sind bisher nur einzelne Ansätze erkennbar, eine verstärkte Einbeziehung tut not.

In einigen Ländern ist inzwischen die Bekämpfung des Gewaltverhaltens der Täter durch Angebote von speziell auf die Thematiken bei häuslicher Gewalt zugeschnittenen, standardisierten Verhaltenstrainings für Täter („Täterarbeit“) etabliert und wird zum Teil flächendeckend gefördert. Sehr hilfreich erscheint in diesem Zusammenhang die im September verabschiedete Reform der Strafprozessordnung ( $\$ 153$ a StPO) und der Änderung des StGB in Zusammenhang mit den Auflagen bei der Verwarnung mit Strafvorbehalt. Danach ist nunmehr mit den neuen Fristenregelungen und der ausdrücklichen Verankerung der Auflagen im Bereich der Einstellung und der Aussetzung der ausgesprochenen Geldstrafe zur Bewährung die Umsetzung eines Verhaltenstrainings Täterarbeit gesetzeskonform möglich und sinnvoll, wie es bereits seit längerer Zeit von Opfern wie auch Tätern häuslicher Gewalt gewünscht wurde.

Der djb hält einen flächendeckenden Ausbau dieses hochwirksamen Instruments zur Bekämpfung häuslicher Gewalt überall für unverzichtbar.

Zusammenfassung:

http://www.djb.de/static/common/download.php/save/1218/ st12-9a_GewaltschutzDok.pdf

\title{
Gewaltschutz - die Rechte der Kinder
}

\section{Susanne Köhler}

Vorsitzende der Fachkommission Gewalt gegen Frauen und Kinder des djb; Rechtsanwältin, Dresden

Bei häuslicher Gewalt gegen ein Elternteil sind Kinder immer (mit-)betroffen: Häufig erleiden sie selbst Gewalt oder beobachten sie. In jedem Fall hat dies schädigende Folgen auf die psychische Gesundheit und die Entwicklung des Kindes. Wie sich aus der Studie des BMFSFJ zu Gewalt gegen Frauen aus dem Jahr 2004 ergibt, berichteten 60 Prozent der befragten Frauen über die letzte gewaltbelastete Paarbeziehung, dass in dieser Paarbeziehung auch Kinder lebten, 57 Prozent der Befragten gaben an, die Kinder hätten die gewalttätigen Situationen gehört, 50 Prozent führten aus, die Kinder hätten die Gewaltsituationen auch selbst beobachtet. Nach Angaben der betroffenen Frauen wurde jedes zehnte Kind selbst körperlich angegriffen. ${ }^{1}$ Dabei hat jedes Kind das Recht auf gewaltfreie Erziehung, $\mathbb{S} 1631$ II BGB.

Um die juristischen Möglichkeiten des Eingreifens und des Schutzes für von Gewalt betroffene Kinder zu erfassen, muss man sich zunächst das zivilrechtliche Rechtssystem der elterlichen Sorge und des Umgangsrechtes vor Augen führen.
Zur elterlichen Sorge gehören zwei Hauptfelder: Die Personensorge, die die Bestimmung über den Aufenthalt, die gesundheitlichen Belange, die schulischen Belange etc. erfasst sowie die Vermögenssorge, $\mathbb{S} \mathbb{S} 1626$ ff. BGB.

Für das Umgangsrecht gilt: Umgang mit beiden Elternteilen entspricht in der Regel dem Wohl des Kindes, so $\mathbb{S} 1626$ III BGB, dabei sind die Eltern zum Umgang berechtigt und

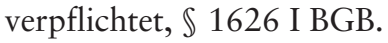

Gerichtliche Eingriffsmöglichkeiten in die elterliche Sorge erfolgen dabei auf Antrag etwa des mitsorgeberechtigten Opfers auf alleinige elterliche Sorge gemäß $\$ 1671$ BGB oder aber aufgrund einer Anregung des Jugendamtes und/oder Dritter, aber auch aufgrund von Kenntnissen des Gerichtes - so etwa im Rahmen eines Kontaktverbotsverfahrens nach $\mathbb{} 1$ Gewaltschutzgesetz - mit eigener Veranlassung gemäß $\mathbb{S} 1666$ und 1666a BGB.

Voraussetzungen für einen Eingriff gemäß $\mathbb{S} 1666$ BGB ist eine bereits eingetretene und fortwirkende oder eine gegenwärtige Gefahr des Kindeswohls, sodass sich bei weiterer

1 So BMFSFJ (Hrsg.): Lebenssituation, Sicherheit und Gesundheit von Frauen, 2004, <http://www.bmfsfj.de/BMFSFJ/Service/Publikationen/publikationen,did=120792.html $>$ (Zugriff: 15.1.2013). 
Entwicklung eine erhebliche Schädigung des Kindes mit großer Sicherheit vorhersehen lässt. Inhaltlich ist der Eingriff auf dasjenige zu begrenzen, was notwendig ist. Es sind stets mildere Maßnahmen wie Gebote, Verbote oder auch Teilentzug der elterlichen Sorge zu ergreifen. ${ }^{2}$

Typischer Grund für den Entzug der elterlichen Sorge ist etwa Drogensucht eines Elternteils. ${ }^{3}$ Die Verbüßung einer Strafhaft und die damit einhergehende Erschwernis der Kommunikation der Eltern allein genügt nicht, ${ }^{4}$ ähnlich wird bei größerer Entfernung der Wohnorte der Elternteile entschieden. Der BGH hat im Jahr 2007 in einem Fall, in dem die Kindesmutter trotz negativer Beweisaufnahme den Verdacht gegenüber dem Vater wegen Kindesmissbrauchs nicht aufgeben kann, dieser die alleinige elterliche Sorge übertragen. ${ }^{5}$ Schon im Jahr 1999 hat der BGH festgestellt, dass keine gesetzliche Vermutung dafür besteht, dass die gemeinsame elterliche Sorge nach der Trennung der Eltern im Zweifel die für das Kind beste Form der Wahrnehmung elterlicher Verantwortung ist. ${ }^{6}$

Auch das Umgangsrecht wird bestimmt durch das Kindeswohl. Es kann derart beschränkt werden, dass nur noch begleiteter und/oder stundenweiser Umgang zu gewähren ist oder das Umgangsrecht befristet ausgeschlossen wird.

\section{Was bedeutet „Kindeswohl“"?}

Leider existiert keine Definition des Begriffes. Er unterliegt als unbestimmter Rechtsbegriff der Auslegung durch das Gericht anhand von entwickelten Kindeswohlkriterien. In der UNKinderrechtekonvention wird u.a. auch vom Recht auf eine Familie, elterliche Fürsorge und ein sicheres Zuhause und vom Recht auf Gesundheit gesprochen.

Nach Artikel $1 \mathbb{S} 1$ des Bundeskinderschutzgesetzes ist es eine staatliche Aufgabe, „das Wohl von Kindern und Jugendlichen zu schützen und ihre körperliche, geistige und seelische Entwicklung zu fördern“. In $\mathbb{} 1$ Absatz 2 heißt es weiter: „Pflege und Erziehung der Kinder und Jugendlichen sind das natürliche Recht der Eltern und die zuvörderst ihnen obliegende Pflicht. Über ihre Betätigung wacht die staatliche Gemeinschaft"; in $\mathbb{} 1$ Absatz 3 heißt es: „Aufgabe der staatlichen Gemeinschaft ist es, soweit erforderlich, Eltern bei der Wahrnehmung ihres Erziehungsrechts und ihrer Erziehungsverantwortung zu unterstützen “.

An dieser Stelle ist das Jugendamt gefragt, das gemäß $\int 8$ a SGB VIII einen Schutzauftrag bei Kindeswohlgefährdung hat und gemäß $\mathbb{8} 8$ SGB VIII fachliche Beratung und Begleitung zum Schutz von Kindern und Jugendlichen sicherzustellen hat. Gemäß $\ 42$ SGB VIII kann auch eine Inobhutnahme erfolgen, so wenn das Kind oder die/der Jugendliche um Obhut bittet oder eine dringende Gefahr für das Wohl des Kindes oder der/des Jugendlichen die Inobhutnahme erfordert.

Wichtige Kriterien des Kindeswohls sind

- Bindungsprinzip (auch Bindungstoleranz): Bindungstoleranz bedeutet dabei die Fähigkeit und Bereitschaft getrenntlebender Eltern, die Beziehung des anderen Elternteils zum gemeinsamen Kind zu respektieren und zu fördern.
- Förderungsprinzip: Pflege, Betreuung, Versorgung und Erziehung.

- Kontinuitätsprinzip: d.h. weitestgehender Erhalt der bisherigen Lebenssituation des Kindes.

- Bindung des Kindes und Kindeswille.

Das Kindeswohl ist immer bei einer missbräuchlichen Ausübung der elterlichen Sorge verletzt, sei es durch die Vernachlässigung des Kindes, unverschuldetes Versagen der Eltern, Kindeswohlgefährdung durch das Verhalten Dritter, mangelnden Wille oder mangelnde Fähigkeit der Eltern zur Gefahrenabwehr oder aber durch häusliche Gewalt.

Besondere Problemfelder in der zivilrechtlichen Praxis bei Gewaltschutzfällen sind dabei die Erwartungen an das Elternteil, welches Opfer ist, im Hinblick auf die Bindungstoleranz sowie die Feststellung des Kindeswillens.

Bindungstoleranz setzt allerdings zum einen eine gleichberechtigte Elternschaft voraus, an der es in Gewaltschutzfällen krankt, zum anderen auch gegenseitigen Respekt, an dem es dem Täter meist fehlt, das Machtgefälle zu Lasten des Opfers ist durch die Intervention nicht sofort aufgehoben.

„Fehlende Kompromissbereitschaft seitens des Gewalt erleidenden Elternteils sollte nicht vorschnell als mangelnde Kooperation gewertet werden, sondern vor dem Hintergrund der erlittenen Gewalt und der schädigenden Auswirkungen auf die Kinder daraufhin überprüft werden, ob sie eine nachvollziehbare Reaktion darstellt. “8 Das Opfer befindet sich im Übrigen in einer zwiespältigen Lage. Es soll dem Kind, welches die gewalttätigen Auseinandersetzungen (mit)erlebt hat, einen Täter als guten Elternteil vermitteln.

\section{Kindeswohl und Kindeswille}

Das Verfahrensrecht des FamFG versucht dem Kindeswillen insoweit gerecht zu werden, als dass gemäß \ 159 FamFG das Kind ab Vollendung des 14. Lebensjahres gerichtlich anzuhören ist, bei Kindern unter 14 erfolgt die Anhörung, wenn Wille und Neigungen von Bedeutung sind. Gemäß der Entscheidung des OLG Brandenburg vom 22. Juli 2010, 9 WF 95/10, ist der Wille eines unter Dreijährigen nicht verlässlich feststellbar. In seiner Entscheidung vom 20. Juli 2010, 10 UF 188/09, führt das OLG Brandenburg aus, dass der Wille eines Achtjährigen nicht allein ausschlaggebend sein kann.

Ferner ist durch das Gericht für eine kindgerechte Information über die eigenen Rechte Sorge zu tragen, meist wird auch ein Verfahrensbeistand bestellt. Dieser soll frühzeitig einbezogen werden und hat ein Recht zur Einlegung von Rechtsmitteln, er gilt als „Vertreter“ des Kindes gemäß $\$ 158$ FamFG. Dabei hat z.B. das OLG Köln, 8. Dezember 2010, 12

2 BVerfG, Beschluss v. 28.2.2012, 1 BwR 3116/11.

3 OLG Bremen, Beschluss v. 21.3.2011, Az. 4 UF 31/11.

OLG Naumburg, Beschluss v. 17.2.2003, Az. 8 UF 189/02.

BGH, Beschluss v. 12.12.2007 - XII ZB 158/05.

BGH, Beschluss v. 29.9.1999, Az. XII ZB 3/99.

7 Gesetz zur Stärkung eines aktiven Schutzes von Kindern und Jugendlichen, Bundeskinderschutzgesetz - BKiSchG v. 22.12.2011.

8 Aus BMFSFJ (Hrsg.): Empfehlungen der Bund-Länder-Arbeitsgruppe „Häusliche Gewalt“ Unterarbeitsgruppe FGG-Reform, 2011, S. 17. 
UF 84/10, beispielsweise entschieden, dass keine gesonderte Anhörung eines Kindes zu erfolgen hat, soweit der Verfahrensbeistand guten Kontakt zum Kind hat.

Verfahrensrechtlich ist eines der Hauptprobleme die Nachweisbarkeit der Kindeswohlgefährdung durch den „Täterelternteil“. Aber auch die Beweisbarkeit der Übergriffe auf das Opfer ist wegen fehlender Zeugen problematisch, ebenso die Beweisbarkeit der Beeinflussung der Kinder durch den Täter. $\mathrm{Zu}$ all diesen Problemfeldern liegen meist nur Einzelfallentscheidungen der Oberlandesgerichte vor.

Bei einmaliger oder nur „geringfügiger“ Körperverletzung, Taten ohne Anwesenheit der Kinder, der „nur“ verbalen Herabwürdigung im Beisein der Kinder wird meist an der gesetzlich normierten Regel der gemeinsamen elterlichen Sorge festgehalten oder aber auf eine vergleichsweise Lösung, die in Gewaltschutzverfahren nach $\mathbb{S} \mathbb{S} 1$ und 2 GewSchG gerade nicht gewollt ist, so $\mathbb{} 36$ I FamFG, hingewirkt.

Häufig wird das Opfer als nicht glaubwürdig oder aber begrenzt erziehungsfähig eingeschätzt, wenn es mehrfach zu Trennung und Rückkehr in den gemeinsamen Haushalt gekommen ist.

Das OLG Köln hat in seiner Entscheidung vom 22. März $2011 \mathrm{zu} 4$ UF 29/11 ausgeführt, dass offen ausgetragene Elternkonflikte eine störungs- und angstfreie Entwicklung der Kinder verhindern; da in dem zu entscheidenden Fall eine Besserung auch in Zukunft nicht erwartet werden könne, wurde beiden Elternteilen die elterliche Sorge entzogen: „Auf Seiten des Kindesvaters fielen dabei verstärkend gravierende persönliche charakterliche Defizite ins Gewicht. So scheint der Kindesvater nicht in der Lage zu sein, seine Emotionen in den Griff zu bekommen. Vielmehr neigt er auch in Gegenwart der Kinder zu verbalen Ausfällen und auch Tätlichkeiten zumindest gegenüber der Antragsgegnerin. Dies hat wiederholt zur Flucht der Antragsgegnerin in Frauenhäuser geführt. Andererseits ist auch die Antragsgegnerin nicht in der Lage, Konstanz und Kontinuität in die Erziehungstätigkeit zu bringen. Auch sie ist viel zu sehr in der Auseinandersetzung mit ihrem Ehemann verhaftet, als dass sie das Wohl ihrer Kinder im Auge hätte. Es ist nicht absehbar, dass sich diese Konflikte trotz ihrer negativen Auswirkungen auf das Bedürfnis der Kinder nach Sicherheit und Stabilität beenden ließen. “9

Demgegenüber hat das OLG Saarbrücken am 30. Juli 2010, 6 UF 52/10, die elterliche Sorge auf das Opfer als alleinberechtigtes Elternteil übertragen, dieses verweigerte wegen der erheblichen Gewalttätigkeit des anderen die Kommunikation mit dem Täter.

Betreffs des Umgangsrechtes ist die Entscheidung des OLG Köln 7. Dezember 2010, 4 UF 183/10, herauszustellen; es führt aus, dass es in Fällen schwerer häuslicher Gewalt und hierdurch schwer traumatisierter, die Gewalt miterlebender Kinder gerechtfertigt ist, das Umgangsrecht des Täters auf briefliche Kontakte und eventuelle Bildinformationen zu beschränken. Das OLG Hamm sprach einen befristeten Umgangsausschluss von zwei Jahren bei Besorgnis einer erneuten Kindesentführung aus, so am 10. Mai 2010, II 6 WF 184,
09. Vom OLG Rostock wurde mit Beschluss vom am 7. Mai 2009, 10 UF 33/09, ein befristeter Umgangsausschluss von drei Monaten für gerechtfertigt gehalten.

Für Gewaltschutzfälle bedeutsam ist die Entscheidung des OLG Düsseldorf vom 27. März 2009, 6 UF 191/08. Verweigert etwa ein elfjähriges Kind nachdrücklich aufgrund eigener nachvollziehbarer Entscheidung den Umgang mit dem nicht sorgeberechtigten Elternteil, kann der sorgeberechtigte Elternteil nicht durch eine Zwangsgeldandrohung gezwungen werden, erzieherisch auf die Entscheidung des Kindes Einfluss zu nehmen.

Zivilrechtlicher Schutz vor (weiterer) häuslicher Gewalt steht damit im ständigen Zwiespalt zwischen Kindeswohl und Recht des „Täterelternteils“ auf Erhalt des Rechts auf elterliche Sorge und umfassenden Umgang.

\section{Was wäre zu verbessern?}

In der gerichtlichen Praxis, aber auch der Beratungspraxis der Jugendämter, wird bei Gewaltschutzfällen immer wieder als Argument für den Erhalt der gemeinsamen elterlichen Sorge und eines umfassenden Umgangs des Kindes mit dem Täter das Entfremdungsrisiko angeführt. Aus Blickrichtung des durch häusliche Gewalt (mit-)betroffenen Kindes bedeutet dies eine fortgesetzte Traumatisierung. Die Beendigung dieser Traumatisierung muss dem möglichen „Entfremdungsrisiko“ vorgehen. Auch im Rahmen der elterlichen Sorgeverfahren bzw. Umgangsverfahren muss der Schutzgedanke vor Kooperationsverpflichtung und gütlicher Einigung stehen.

\section{Aufhebung des $\S 3$ Gewaltschutzgesetz: eine Alternative?}

Weitergehend ist zu fragen, ob der über $\$ 1666$ a BGB intendierte Schutz für von häuslicher Gewalt (mit)betroffene Kinder wirklich ausreichend ist.

In der Diskussion steht, Kinder und Jugendliche nicht mehr aus dem Schutz der Paragrafen des Gewaltschutzgesetzes herauszunehmen und damit diesen das Recht einzuräumen, eigene Anträge auf Kontaktverbot oder Wohnungszuweisung zu stellen. Bisher schließt $\mathbb{S}$ GewSchG dies aus. Lediglich der betroffene Elternteil kann sich selbst vor direktem Kontakt mit dem Täter schützen, das betroffene Kind kann jedoch nur indirekt durch ein Annäherungsverbot an die Wohnung, die Schule, die Kindertageseinrichtung oder ähnliches geschützt werden.

Befürworter/innen der Aufhebung des $\mathbb{\$} 3 \mathrm{GewSchG}$ sehen darin die Möglichkeit der kurzfristigen Entlastung des Sorgebzw. Umgangsverfahrens sowie der Verbesserung der Beweislastsituation, da in den Verfahren nach $\mathbb{S} \mathbb{S} 1666,1666 \mathrm{a}$ BGB nicht die Beweislastumkehr des Gewaltschutzgesetzes zugunsten des Opfers greift. Auch fehlt es aufgrund der Herausnahme der Kinder und Jugendlichen aus dem direkten Schutzbereich des Gewaltschutzgesetzes an der besonderen opferentlastenden Strafbewährung eines Verstoßes gegen die richterliche Anordnung gemäß $\mathbb{4}$ GewSchG.

9 S. <http://www.justiz.nrw.de/nrwe/olgs/koeln/j2011/4 UF_29_11beschluss20110322.html> (Zugriff: 8.1.2013). 
Die Notwendigkeit einer Aufhebung wird im Weiteren damit begründet, dass diese faktische Schlechterstellung der von häuslicher Gewalt (mit-)betroffenen Kinder gegenüber den betroffenen Opfern vor allem gegen die Grundrechte auf Gleichbehandlung in Artikel 3 GG und auf körperliche Unversehrtheit in Artikel 2 GG verstößt.

Für die Beibehaltung des $\mathbb{3}$ GewSchG in bisheriger Form wird angeführt, dass die Erweiterung der rechtlichen Mög- lichkeit sich als zusätzliche Belastung für das Kind auswirken kann, welches durch ein eigenes Antragsrecht noch mehr in einen Loyalitätskonflikt gebracht würde und dann auch zusätzlich bewusster, aber auch unbewusster Beeinflussung der Elternteile ausgesetzt werden könnte.

Diese Diskussion bleibt im Auge zu behalten und Für und Wider fundiert miteinander abzuwägen.

\section{Häusliche Gewalt - und was ist mit den Kindern?'}

\section{Sabine Heinke \\ Aufsichtsführende Richterin am Amtsgericht, Bremen}

Wir wissen mittlerweile, dass Gewalt in Paarbeziehungen nicht „aus heiterem Himmel“ auftritt, sondern die Folge eines erlernten Verhaltens darstellt, welches über kurz oder lang zur Anwendung kommt. Es handelt sich häufig um ein über Generationen weitergegebenes Handlungsmuster. Sowohl Täter als auch verletzte Person haben regelmäßig im familiären Umfeld Erfahrungen machen müssen, die Gewalt für sie als Machtmittel in der Beziehung erscheinen lässt, das man anwenden kann bzw. hinnehmen muss. Kinder lernen durch das Beispiel, das ihre Eltern durch ihr Verhalten geben. Gewalt als Mittel der familiären Kommunikation und Strukturgestaltung ist ein Lerninhalt, den Kinder übernehmen, selbst wenn sie die Folgen dieses Handelns für sich ablehnen, ganz einfach deshalb, weil es ihnen mangels anderweitiger Erfahrung an Handlungsalternativen fehlt. Es gilt als sicher, dass das Miterleben von Gewalt in der Herkunftsfamilie überproportional häufig in der nächsten Generation Gewalttäter oder Opfer hervorbringt. Wer als Kind sehen, hören oder fühlen musste, wie ein Elternteil den anderen erniedrigt, geschlagen, bedroht und verletzt hat, erwirbt häufig nur ein geringes Selbstwertgefühl und hat ein höheres Risiko, psychische Auffälligkeiten bis hin zu Suchterkrankungen zu entwickeln. Ein Kind, das gewalttätigem Handeln zwischen seinen Eltern ausgesetzt war, kann hiervon dauerhafte Ängste und schwere Traumata zurückbehalten. ${ }^{2}$ Zuweilen wird man nicht umhinkommen, sich vorzustellen, was man fühlen würde, wenn Vater und Mutter laut streiten; wenn man sieht oder hört, wie geschlagen wird, welche Gefühle von Angst, Panik, Hilflosigkeit, Wut, Entsetzen in der Person entstehen, die dieses Handeln miterlebt. ${ }^{3}$ Im Kindesalter erlebbare Folgen dieser emotionalen Belastungen sind Verhaltensauffälligkeiten, Lernstörungen, Entwicklungsverzögerungen aller Art.

Wie bereits an anderer Stelle 4 ausgeführt, sind die Auswirkungen des gewalttätigen Handelns in der Familie damit nicht ausreichend beschrieben. Die gewalttätigen Übergriffe eines Partners gegen die/den andere/n haben noch viele andere Implikationen: Da ist zunächst der Umstand, dass Erwachsene, die sich derart tätlich angreifen und streiten, sich an ihrem Tun nicht davon hindern lassen, dass ihre Kinder dies miterleben und unter den Folgen dieses Tuns leiden werden. Das heißt, es fehlt, jedenfalls dem Täter, zuweilen auch der verletzten Person, an Empathie und an Verantwortungsbewusstsein. Die eigene Befindlichkeit als Täter oder Opfer wird deutlich vor die der Kinder gestellt. Häufig werden die Kinder im Machtkampf der Eltern instrumentalisiert, eine Parentifizierung tritt ein, die Kinder müssen dem Opfer-Elternteil zur Seite stehen; immer eine deutliche Überforderung der Kinder. Andererseits zeigt sich immer wieder eine Identifizierung mit dem starken, mächtigen, Gewalt ausübenden Elternteil und eine Verachtung des schwachen, der Gewalt unterworfenen, Elternteils. Beides bedeutet, dass die Erziehungsfähigkeit der Eltern gegenüber ihrem Kind nicht mehr gegeben ist, wie auch die zuvor geschilderten Folgen des gewalttätigen Handelns für das familiäre Zusammenleben und die Familienstruktur sämtlich Auswirkungen auf die Erziehungsfähigkeit der Eltern haben, gleichzeitig aber auch auf die Akzeptanz der Kinder, auf ihre Bereitschaft, den Eltern zu vertrauen, auf sie zu hören, die ebenso schwindet wie der Glaube daran, dass die Eltern Schutz und Sicherheit bieten könnten. Die Gewaltausübung in der Familie untergräbt nicht nur das Verhältnis der Kinder zu dem Gewalt ausübenden Elternteil, sondern auch zu der „verletzten Person“. 5

Das Betroffensein ${ }^{6}$ von Partnerschaftsgewalt im nahen sozialen Umfeld hat grundsätzlich die Qualität einer erstrangigen

1 Dieser Beitrag sollte ursprünglich als Diskussionsgrundlage für den Fachkongress des djb: 10 Jahre Gewaltschutzgesetz - neue Herausforderungen dienen und anhand der Diskussionsbeiträge überarbeitet werden. Da der Vortrag wegen Krankheit der Referentin ungehalten blieb, fehlen leider die von ihr erwünschten und erwarteten Diskussionsbeiträge, insoweit enthält der Beitrag vorläufige Problembeschreibungen. Ich danke Susanne Köhler herzlich, dass sie meine Aufgabe übernommen hat.

2 Zu den Auswirkungen z.B. Ostbomk-Fischer, Elke, Das Kindeswohl im Ernstfall, KindPrax 2003, 8; verschiedene Veröffentlichungen von Kindler, Heinz u.a., z.B. Auswirkungen von häuslicher Gewalt auf die Entwicklung von psychosoziale Entwicklung von Kindern, FPR 2005, S. 16 ff.

3 Nicht vergessen werden sollte auch, dass das Quälen nahestehender Personen vor den Augen der Angehörigen schon immer eine wirkungsvolle Foltermethode darstellte.

4 Heinke, Sabine, Umgangsrecht und Partnerschaftsgewalt - nicht nur ein mechanisches Problem, STREIT 2008, S. 3 ff.

5 Vgl. ebd.; Literaturhinweise bei NK-BGB/Heinke, Sabine § 3 GewSchG. 